\title{
Antibodies from combinatorial libraries use functional receptor pleiotropism to regulate cell fates
}

Richard A. Lerner ${ }^{1,2} *$, Rajesh K. Grover ${ }^{1}$, Hongkai Zhang ${ }^{1}$, Jia Xie ${ }^{1}$, Kyung Ho Han ${ }^{1}$, Yingjie Peng ${ }^{1}$ and Kyungmoo $\mathrm{Yea}^{3}$

\footnotetext{
${ }^{1}$ Department of Cell and Molecular Biology, The Scripps Research Institute, La Jolla, CA 92037, USA

${ }^{2}$ Department of Chemistry, The Scripps Research Institute, La Jolla, CA 92037, USA

${ }^{3}$ Shanghai Institute for Advance Immunological Studies, ShanghaiTech University, Shanghai 200031, China

Quarterly Reviews of Biophysics (2015), 48(4), pages 389-394 doi:10.1017/S0033583515000049
}

Abstract. To date, most antibodies from combinatorial libraries have been selected purely on the basis of binding. However, new methods now allow selection on the basis of function in animal cells. These selected agonist antibodies have given new insights into the important problem of signal transduction. Remarkably, when some antibodies bind to a given receptor they induce a cell fate that is different than that induced by the natural agonist to the same receptor. The fact that receptors can be functionally pleiotropic may yield new insights into the important problem of signal transduction.

Key words: Combinatorial Libraries, Pleiotropism, Cell Fates Regulations.

\section{Introduction}

Biological and physical diversity has long fascinated scientists and non-scientists alike. For instance, the stripes on no two zebras are alike nor is any snowflake exactly like another. We have been interested in using the chemical diversity of the immune system to generate reagents for research and therapy. Our tool has been the combinatorial antibody library (Barbas et al. 1991, 1992; Hoogenboom \& Winter, 1992; Hoogenboom et al. 1991; Huse et al. 1989; Kang et al. 1991; Lerner, 2006; Lerner et al. 1992; Marks et al. 1991; McCafferty et al. 1990; Orlandi et al. 1989; Sastry et al. 1989). Combinatorial antibody libraries are essentially a chemically synthesized immune system that nowadays has about $1.0 \times 10^{11}$ unique members. This is about three orders of magnitude larger than the antibody repertoire in any individual, yet it is contained in only a few hundred microliters. Thus, today scientists have control over $10^{11}$ unique binding proteins. Since their inception these libraries have served the scientific community well both for research purposes

* Authors for correspondence: R. A. Lerner, Department of Cell and Molecular Biology, The Scripps Research Institute, La Jolla, CA 92037, USA. Tel.: 858-784-8265; Fax: 858-784-2791; E-mail: rlerner@scripps.edu and human therapy. For instance, the largest selling drug in the world, Humira, was generated from a combinatorial antibody library. However, recently these libraries have been converted into a format that renders them infectious for animal cells thereby giving them the potential to regulate cell fates. Thus, we can think of the research potential of this methodology as the ability to introduce 100 million different binding proteins into a population of cells such that each cell has one new binding protein. In chemical terms, the experimenter can now alter the binding landscape of cells. In this perspective, we will introduce the general concepts that underlie combinatorial antibody libraries, by way of background, and then turn to the newer intracellular libraries.

\section{Contrasting the normal immune system to the chemical immune system}

One reason combinatorial antibody libraries have been successful is that they use the organizational features of the natural immune system, albeit with much larger numbers 


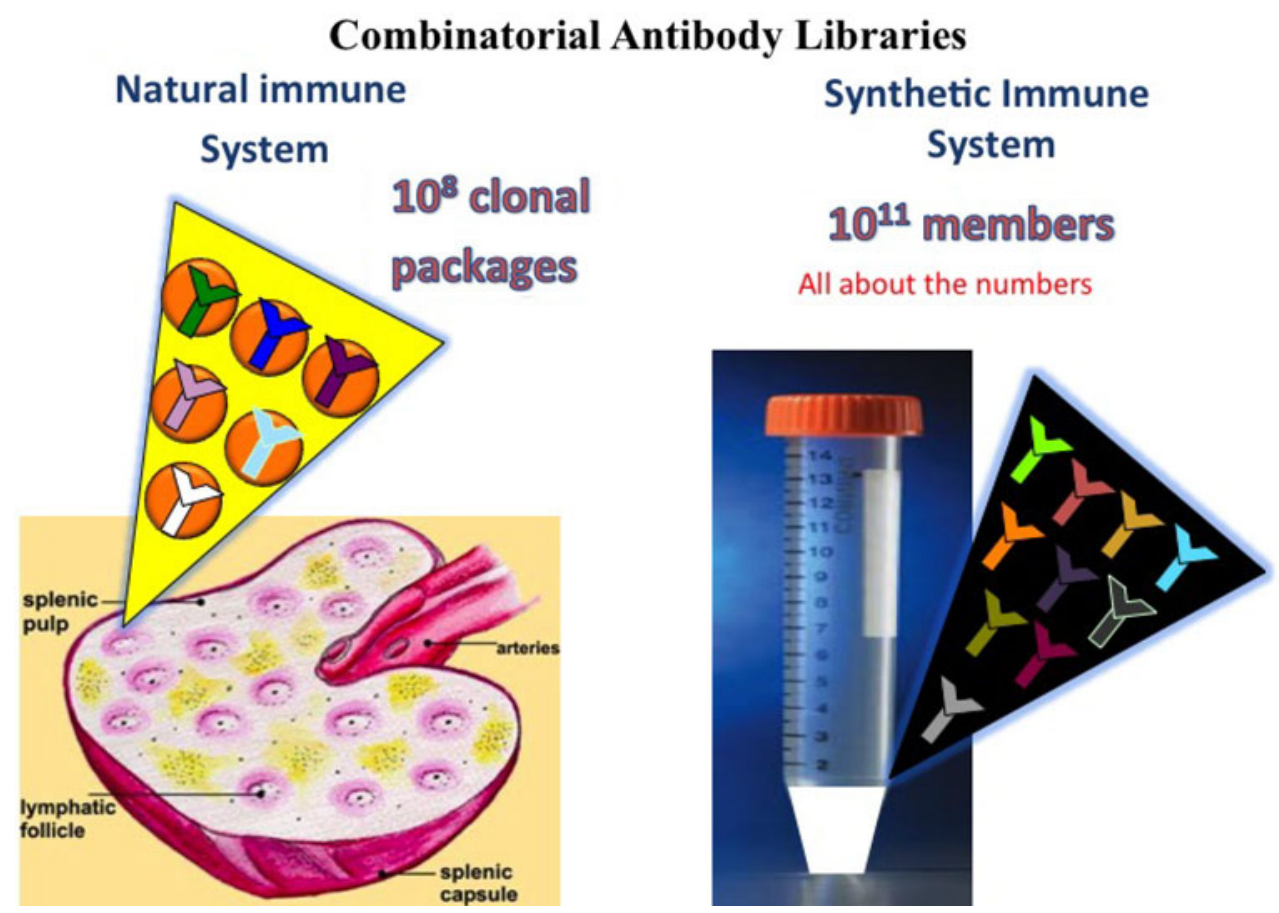

Fig. 1. Comparison of the natural immune to the synthetic version. On the left is an image of a spleen that contains antibodies that are packaged individually in B-cells. This packaging allows recognition and replication to be linked. On the right is a depiction of a synthetic immune where the genes encoding $10^{11}$ different antibody genes are present in solution but not yet packaged.

of starting binding proteins. The most important general feature of the natural immune system is that recognition (of antigen) and replication are linked. This is accomplished by a packaging strategy such that each of the 100 million members of the repertoire is contained in a single cell that when exposed to antigen replicates. Thus, the system is clonal (Fig. 1). By contrast, in the generation of combinatorial libraries, this packaging trick is initially ignored and a vast number of antibody genes are synthesized. Thus, cloning takes complexity and reduces it to a singularity whereas, initially, combinatorial libraries convert the clonal singularities of the natural immune system to complexity. Thus, if one is to adopt the strategy of the immune system, complexity must be converted back to singularities. Operationally, this reduces to the fact that one has about $10^{11}$ antibody genes in solution and one must repackage them like in the immune system to link recognition and replication. There are many ways to do this, but they all share the feature that the antibody genes are inside a replicating organism and the encoded antibody is on the outside (Fig. 2). Thus, like the natural antibody repertoire each antibody is packaged separately and recognition and replication are linked. In Fig. 3, we visualize the M-13 page system using an antibody to the Hepatitis B particle. The hepatitis B antigen is chosen as an example simply because it is visible in the electron microscope. The M-13 phage is $1 \mu \mathrm{m}$ long rod that has been engineered to, on the average, express one antibody molecule per phage. Note that the phage binds to the antigen at their tips. This is because the antibody is incorporated into the gene 3 protein that is located on the tip of the phage. Thus, these libraries allow creation of a synthetic immune system. They use the principles of the natural repertoire where genotype and phenotype are linked in a package capable of replication. But, they have important advantages over the natural immune system in that they eliminate the century old tradition of immunization or, indeed, the use of live animals. Finally, they circumvent the phenomenon of immune tolerance, which is a restriction against making antibodies to self-proteins. This is important because most therapeutic antibodies are against selfantigens. Since the ability to circumvent tolerance is one of the key strengths of the combinatorial antibody libraries, it is perhaps worth explaining a little further for the general reader. In terms of theory and practice, immune tolerance is the centerpiece of immunology. It is most often couched in terms of discrimination of self from non-self. Simply put, it means one cannot make antibodies to one's self. Thus, generally, clones making antibodies to self are eliminated during the development of the immune repertoire in an individual. This process is not perfect and sometimes antibodies against self appear in disease states such as Lupus. Collectively, these diseases are termed autoimmune diseases and can be quite severe or even lethal. However, as mentioned, combinatorial antibody libraries are synthetic and are, thus, not restricted by tolerance that is an intact animal phenomenon that only applies when live animals are used 


\section{First Generation Combinatorial Antibody Library Visualization of an Antibody to Hepatitis B}
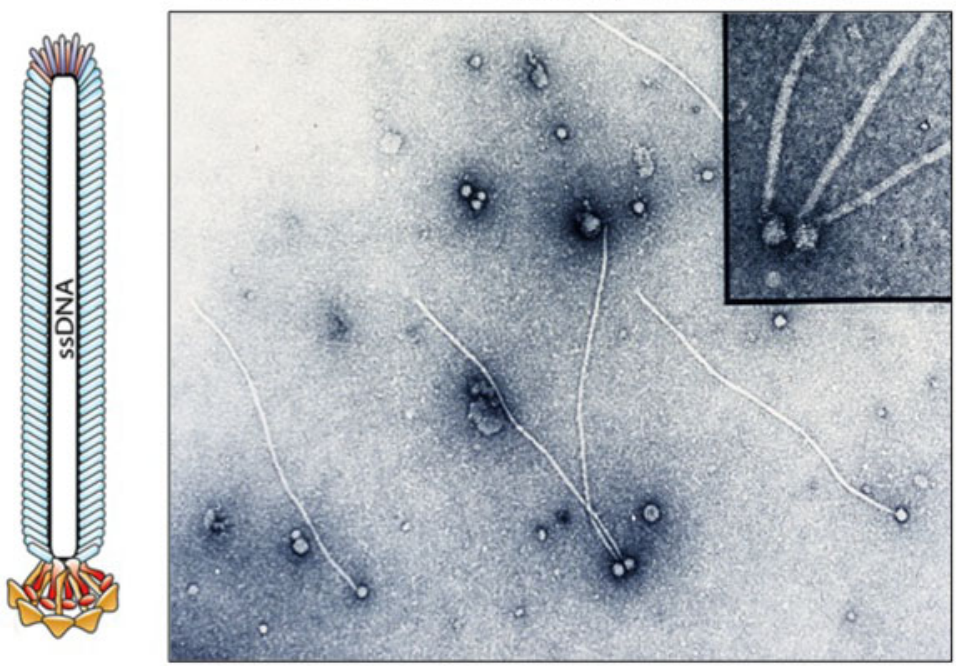

Fig. 2. Visualization of the M-13 phage selection system. In the left is a schematic of a phage particle. The phage is a micron long tube containing ssDNA encoding the antibody in its interior and the encoded antibody protein expressed on its tip as part of the phage encoded gene 3 protein. On the right is an electron micrograph showing the phage particle (rods) attached only at their tips to Hepatitis $\mathrm{B}$ virus (spherical particles). Insert shows higher power magnification of the process. Rarely, two separate phages attach to a virus particle because the particle has many antigenic sites.

during the process of immunization. Since, as mentioned, almost all therapeutic antibodies are used to perturb self-proteins whether they are in the circulation or on the surface of cells, it is important to have a method where very large numbers of fully human anti-self antibodies can be selected. This is especially true for agonist antibodies, because functional antibodies can be rare and one needs large numbers of input antibodies to select the most potent ones. As will be seen below, after selection for binding, one may have as many as 100000 anti-self antibodies out of which only two or three are functional. Thus, it is not sufficient to violate the restriction of tolerance, but one must do it many times! One final point, Immunochemistry has entered a phase that requires precision where the issue is not whether you simply bind to a target (antigen), but rather where and how you bind and what is the chemistry of the interaction. This means choice. And, choice is best served by a large and diverse input repertoire that is unrestricted.

So at this point we can summarize where we are. We have access to a very large number of potential binding proteins $\left(10^{8}-10^{11}\right)$. We can select for binding and, if the desired phenotype is simply binding then genotype is already linked to phenotype. But the current goal is to go beyond simple binding and directly link antibody genotype to cellular phenotype!

\section{Direct selection for cellular phenotype}

The approach to the direct selection for phenotype is shown in Fig. 4. The antibody library is moved from an M-13 vector to lentivirus thereby allowing animal cells to be infected with the antibody library which is, for technical reasons, limited to $10^{8}$ members.

(When dealing with animal cells, the size of the input library is only a function of the cell handling capability of the usual academic laboratory. Much larger numbers can and have been used. Also, the process is now being automated, especially in industry).

Nevertheless, one gains a new power that more than makes up for the loss of diversity in that the selection is now autocrine based because the antibody and target are in the same cell. The antibody can be confined to any cell compartment such as the cytoplasm or cell surface and the gene encoding the antibody is integrated in the cellular genome from where it can be recovered by PCR (Fig. 4). If, for example, one wishes to select antibody agonists, a reporter system is constructed. In one of the most powerful iterations, when the cell is activated a fluorescent signal is generated, so that cells with agonist antibodies can be analyzed by fluorescence activated cell sorting. Using modern cell sorters, fluorescent signals can be analyzed at a rate of about 20 million events per hour. This approach has turned out to be very powerful and many new agonists have been selected that regulate cell fates from, for example, stem cells (Fig. 5) (Xie et al. 2013, 2014; Yea et al. 2013, 2015; Zhang et al. 2011, 2012, 2013, 2014).

To ensure that the target specificity of the antibody is as suggested, during the selection one always runs an experiment on the same cells that differ only in that the target is not expressed. The target specificity appears to be absolute in 


\section{Returning the System to Clonality and Enabling Selection by Linking Recognition and Replication}
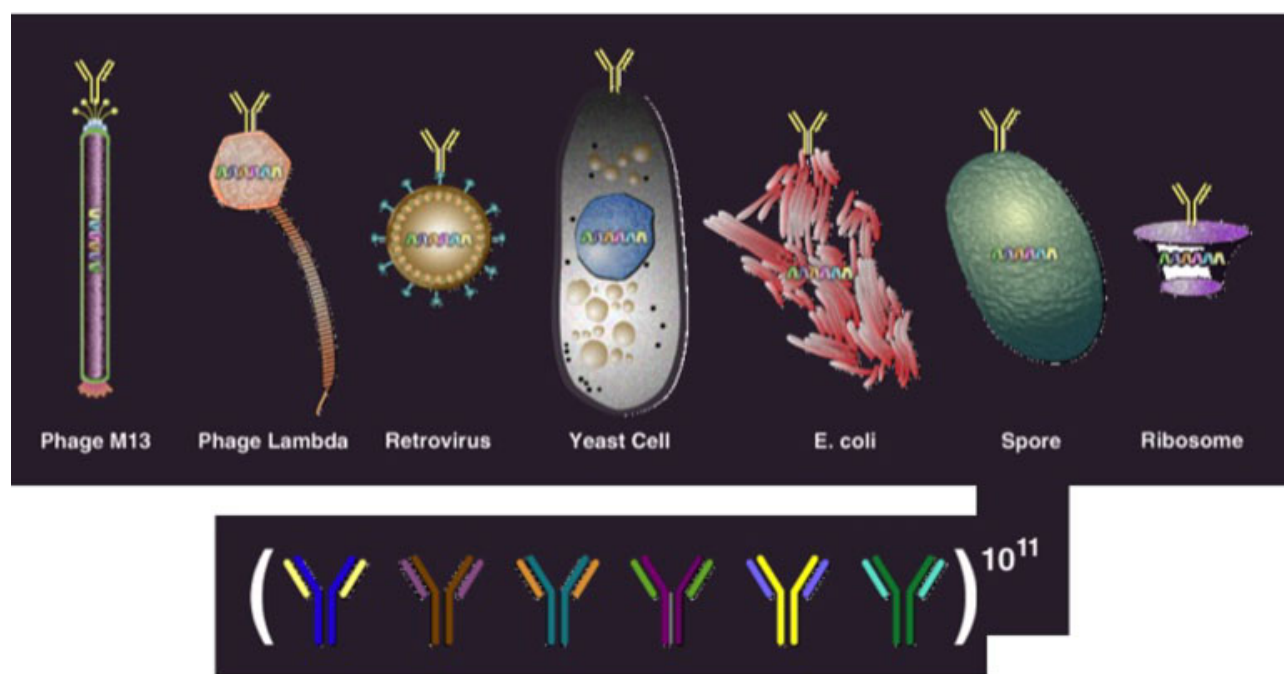

Fig. 3. Methods available to package the antibody genes. In every case except for the ribosome recognition and replication are linked because the gene encoding the antibody is on the inside of an organism capable or replication and the antibody is expressed on the outside. Thus, one can select for binding and replicate all the selected members from a large input antibody library.

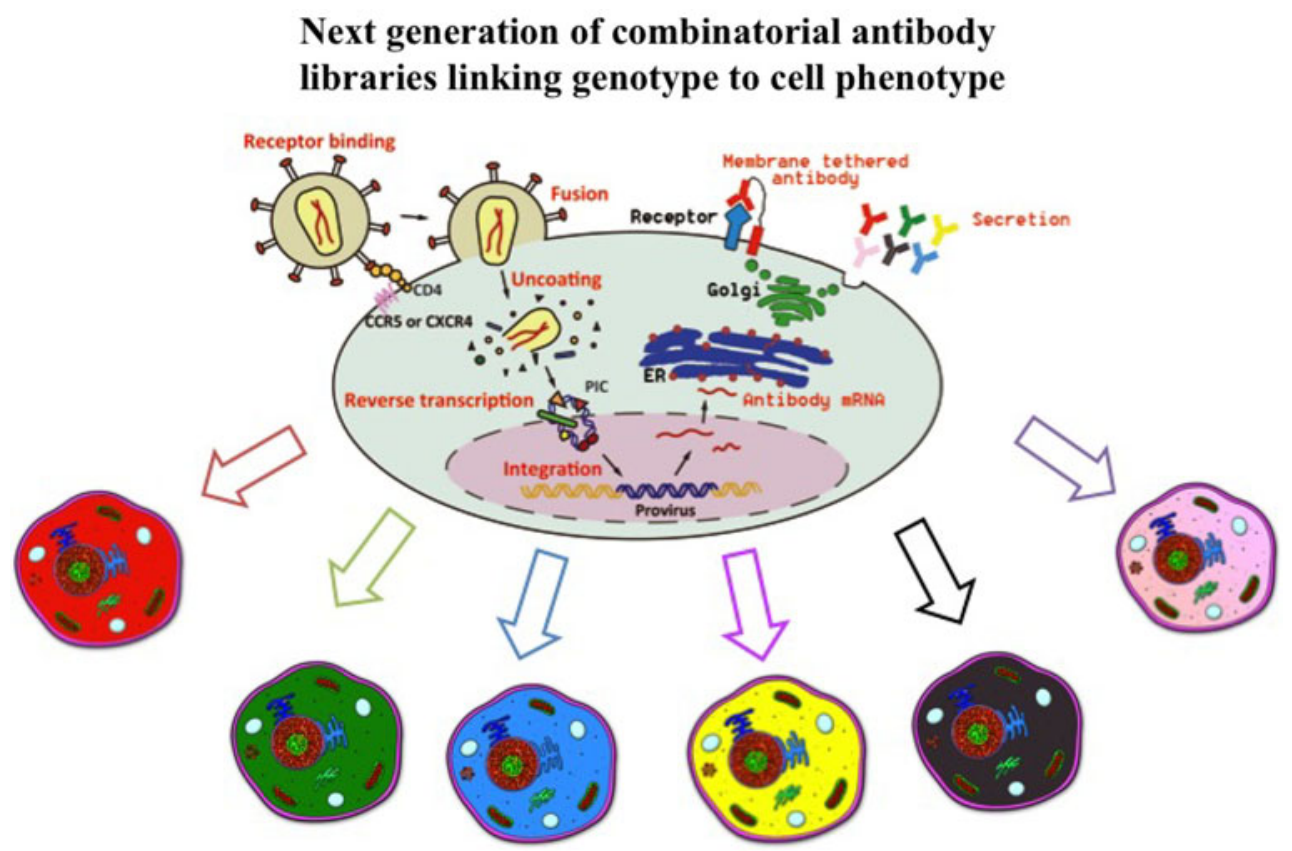

Fig. 4. The method to create intracellular combinatorial antibody libraries. The genes encoding the antibody molecules from a large combinatorial library are inserted into lentiviruses so animal cells can be infected. One the cell is infected the antibody genes are integrated into the cell genome and encode antibody molecules. Such antibodies can be retained in the cytoplasm, secreted, or anchored in the plasma membrane. Some of these expressed antibodies change the differentiation state of the cell. The system is powerful in that it is autocrine because the antibody and its target are expressed in the same cell. Thus, each cell becomes a selection system unto itself. Again genotype and phenotype are linked, but here the linkage is at the level of animal cells themselves. A large variety of selection systems can be used to select from cells where the expressed antibody has induced a new phenotype (Xie et al. 2013, 2014; Yea et al. 2013, 2015; Zhang et al. 2011, 2012, 2013, 2014). Adapted in part from Viral Gene Therapy, book edited by Ke Xu, by authors Suzuki, Y., and Suzuki, Y. Gene regulatable lintiviral vector system. 


\section{Nine Antibodies Selected from Reporter Cells- Nine Different Cell Fates}

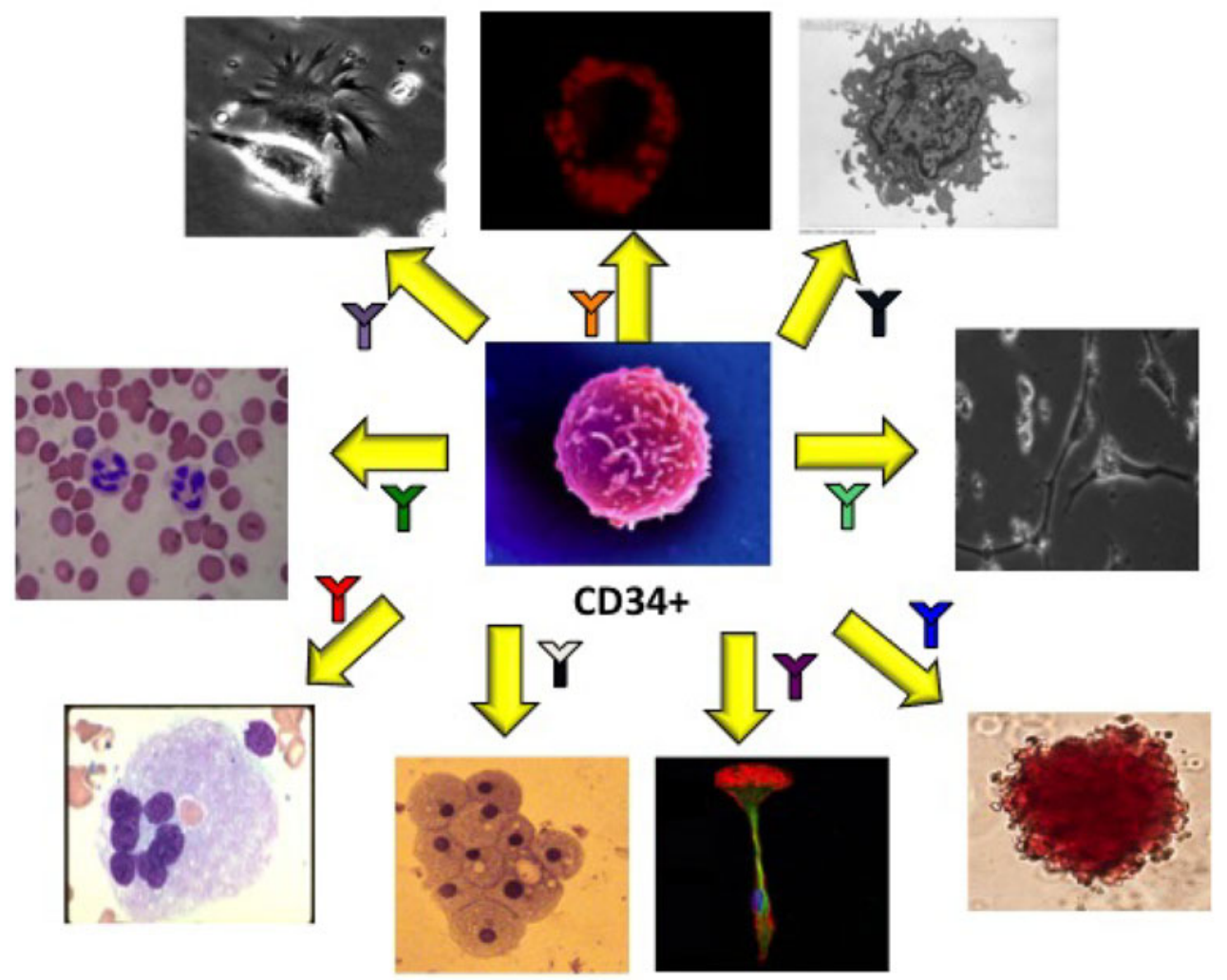

Fig. 5. Many cell fates induced by selected antibodies from a single type of stem cell. CD34 + human stem cells are treated with purified antibodies encoded by genes selected from cells infected with antibody libraries. The induced cell fates are (clockwise from 12:00 o'clock) Brown fat cells, dendritic cells, tendon cells, red cells, neural cells, M2 macrophages, platelets, granulocytes and more mature dendritic cells.

that no phenotypic induction is observed when the target is absent. Please see supporting information figure S6 in reference (Xie et al. 2013) for details of such an experiment.

\section{New teachings}

When one has the ability to introduce 100 million binding proteins into cells, it is reasonable to expect to generate interesting reagents some of which regulate cell fates according to expected mechanisms. However, one also expects to find unusual outcomes that uncover new general principles. One of the most dramatic findings from these intracellular antibodies is the finding that for a given receptor an agonist antibody may induce a cellular fate that is different than that which occurs when the natural agonist binds to the same receptor (Xie et al. 2013). Thus, remarkably, the natural agonist for the granulocyte colony-stimulating factor receptor (GCSFR) induces white cell (granulocyte) formation from CD-34+ human bone marrow cells, whereas an antibody agonist to the same receptor induces neural cells (Xie et al. 2013).
The fact that one receptor can activate two different lineage outcomes is a new paradigm in receptor biology. We term this phenomenon functional receptor pleiotropism, which is a phenomenon that may both provide unique reagents and teach us much about signal transduction. In thinking about how receptor pleiotropism might work one can revisit the general principles of signal transduction because the advent of a new phenomenon can often serve to underscore existing ideas and give new insights. The central idea is that differentiation and development are likely caused by induction of a combinatorial matrix of signaling molecules. Thus, one does not need a very large number of individual molecules to regulate cell fate. It is like generation of diversity in the humoral immune system where one can start with a limited set of genes that ultimately generate huge diversity because of their combinatorial association. Second, in terms of molecular regulatory mechanisms, the differences in pathways leading to vastly different phenotypes may actually be quite subtle and more related than previously thought. Thus, a simple change in the ratio of signal transduction molecules might suffice to change the differentiation pathway. 
An agonist antibody to a given receptor because of affinity bivalency etc. changes the transcription factor matrix resulting in a phenotype that is different than the natural agonist. Thus, the cell is ready to signal but the antibody simply changes the output combination.

\section{Outlook}

Thus, we have seen that combinatorial antibody libraries allow creation of a synthetic immune system in the test tube, which is selectable because recognition and replication are linked. In their new intracellular format, one can select directly for function. The large input diversity allows for selection of rare events.

Importantly, these methods not only allow the generation of research antibodies but, because they circumvent the problem of self-immune tolerance, they are very useful for generation of therapeutic antibodies almost all of which are antibodies to self. Several approved therapeutic antibodies including Humira, which is the largest selling drug in the world, were generated by these methods. Newer results show that in their intracellular format antibody libraries allow for rapid generation of agonist antibodies that are new tools for study of differentiation, development and signal transduction. In the end, one reason that intracellular combinatorial antibody libraries can control cell fates is because they are a gain of function method.

\section{Acknowledgements}

We are grateful for the generous support from the JBP foundation and Zebra Biologics Inc.

\section{References}

Barbas, C. F., Bain, J. D., Hoekstra, D. M. \& Lerner, R. A. (1992). Semi-synthetic combinatorial antibody libraries: a chemical solution to the diversity problem. Proceeding of the National Academy of Sciences of the United States of America 89, 4457-4461.

Barbas, C. F., Kang, A.S., Lerner, R. A. \& Benkovic, S. J. (1991). Assembly of combinatorial antibody libraries on phage surfaces: the gene III Site. Proceedings of the National Academy of Sciences of the United States of America 88, 7978-7982.

Hoogenboom, H. R., Griffiths, A. D., Johnson, K. S., Chiswell, D. J., Hudson, P. \& Winter, G. (1991). Multi-subunit proteins on the surface of filamentous phage: methodologies for displaying antibody (Fab) heavy and light chains. Nucleic Acids Research 19, 4133-4137.

Hoogenboom, H. R. \& Winter, G. (1992). By-passing immunization: human antibodies from synthetic repetoires of germline $V_{\mathrm{h}}$ gene segments rearranged in vitro. Journal of Molecular Biology 227, 381-388.

Huse, W. D., Sastry, L., Iverson, S. A., Kang, A. S., Alting-Mees, M., Burton, D. R., Benkovic, S. J. \& Lerner, R. (1989). Generation of a large combinatorial library of the immunoglobulin repertoire in phage lambda. Science 246, 1275-1281.
Kang, A. S., Barbas, C. F., Janda, K. D., Benkovic, S. J. \& Lerner, R. A. (1991). Linkage of recognition and replication functions by assembling combinatorial antibody fab libraries along phage surfaces. Proceedings of the National Academy of Sciences of the United States of America 88, 4363-4366.

Lerner, R. A. (2006). Manufacturing immunity to disease in a test tube: the magic bullet realized. Angewandte Chemie International Edition in English 45, 8106-8125.

Lerner, R. A., Kang, A. S., Bain, J. D., Burton, D. R. \& Barbas, C. F. (1992). Antibodies without immunization. Science 258, 1313-1314.

Marks, J. D., Hoogenboom, H. R., Bonnert, T. P., McCafferty, J., Griffiths, A. D. \& Winter, G. (1991). By-passing immunization: human antibodies from V-gene libraries displayed on phage. Journal of Molecular Biology 222, 581-597.

McCafferty, J., Grifhiths, A. D., Winter, G. \& Chiswell, D. J. (1990). Phage antibodies: filamentous phage displaying antibody variable domains. Nature 348, 552-554.

Orlandi, R., Gưssow, D. H., Jones, P. T. \& Winter, G. (1989). Cloning immunoglobulin variable domains for expression by the polymerase chain reaction. Proceedings of the National Academy of Sciences of the United States of America 86, 3833-3837.

Sastry, L., Alting-Mees, M., Huse, W. D., Short, J. M., Sorge, J. A., Hay, B. N., JANDA, K. D., BENKOVIC, S. J. \& LeRNER, R. A. (1989). Cloning of the immunological repertoire in Escherichia Coli for generation of monoclonal catalytic antibodies: construction of a heavy chain variable region-specific cDNA library. Proceedings of the National Academy of Sciences of the United States of America 86, 5728-5732.

Xie, J., Yea, K., Zhang, H., Moldt, B., He, L., Zhu, J. \& Lerner, R. A. (2014). Prevention of cell death by antibodies selected from intracellular combinatorial libraries. Chemistry and Biology 21, 274-283.

Xie, J., Zhang, H., Yea, K. \& Lerner, R. A. (2013). Autocrine signaling based selection of combinatorial antibodies that transdifferentiate human stem cells. Proceedings of the National Academy of Sciences of the United States of America 110, 8099-8104.

YeA, K., Xie, J., Zhang, H., Zhang, W. \& Lerner, R. A. (2015). Selection of multiple agonist antibodies from intracellular combinatorial libraries reveals that cellular receptors are functionally pleiotropic. Current Opinion in Chemical Biology 26, 1-7.

Yea, K., Zhang, H., XIE, J., Jones, T. M., Yang, G., Song, B. D. \& LERNER, R. A. (2013). Converting stem cells to dendritic cells by agonist antibodies from unbiased morphogenic selections. Proceedings of the National Academy of Sciences of the United States of America 110, 14966-14971.

Zhang, H., Torkamani, A., Jones, T.M., Ruiz, D. I., Pons, J. \& Lerner, R. A. (2011). Phenotype-information-phenotype cycle for deconvolution of combinatorial antibody libraries selected against complex systems. Proceedings of the National Academy of Sciences of the United States of America 108, 13456-13461.

Zhang, H., Wilson, I. A., \& LerNer, R. A. (2012). Selection of antibodies that regulate phenotype from intracellular combinatorial antibody libraries. Proceedings of the National Academy of Sciences of the United States of America 109, 15728-15733.

Zhang, H., XiE, J., \& Lerner, R. A. (2014). A proximity based general method for identification of ligand and receptor interaction in living cells. Biochemical and Biophysical Research Communications 454, 251-255.

Zhang, H., YeA, K., Xie, J., Ruzz, D., WiLson, I. A. \& Lerner, R. A. (2013). Selecting agonists from single cells infected with combinatorial antibody libraries. Chemistry and Biology 20, 734-741. 